\title{
PREANAESTHETIC FASTING PRACTICE AND OUTCOME : A STUDY IN NEPALI CHILDREN
}

\author{
Maharjan S K*, Bajracharya G R*, Aryal S*
}

\section{ABSTRACT}

The purpose of this study is to compare the outcome of traditionally advised pre-anesthetic fasted children with those who fasted for lesser time in our setup.

One hundred and Sixty two children undergoing surgery under general anaesthesia were selected randomly. They were divided into two groups. Group one was advised in a traditional way - no solid food after midnight and no liquid drink at least six hours before anaesthesia. Group two was given either glucose water 2-4 hours before induction or breast milk 4 hours before induction of anaesthesia. None of the children were premedicated.

Anesthetic techniques were either sole intravenous anaesthesia (IVA) for minor cases or general anaesthesia (GA) and combined methods (IVA or GA with regional blocks). Patients were closely monitored for any active regurgitation and vomiting during the induction of anaesthesia, perioperative and postoperative period. Complications were analyzed in different age groups, different fasting hours and type of anaesthesia delivered.

None of the children had any regurgitation or vomiting during induction and perioperative period. Few children of both groups vomited during postoperative period when they were fully conscious.

As the chances of regurgitation and vomiting with clear fluid given two hours before is comparable with the traditional system, there is no need to put the child starved for prolonged period. This will avoid unnecessary dehydration, hypoglycemia and uncoperation in the children.

\section{Key Words: Pre-anaesthetic fasting, regurgitation, dehydration.}

\section{INTRODUCTION}

Ideally preanesthetic fasting should not produce preoperative dehydration, hypoglycemia or patient discomfort. Prolonged fasting prior to elective surgery is not only uncomfortable to the patient, but has detrimental effects. It causes thirst, hunger and irritability in older children and it may produce dehydration, hypoglycemia and hypovolemia especially in infants and neonates. ${ }^{1}$
It is believed that restriction of liquids and solid food ingestion for specified period for induction of anaesthesia should limit the aspiration and then acid pneumonitis syndrome. But the traditional fast doesn't ensure empty stomach which is shown by various studies done in different centers. A consistent finding is that gastric fluid volume and $\mathrm{pH}$ are independent of the duration of fluid fast beyond 2 hours provided that only clear fluid is ingested on the day of surgery. Hypovolemia

* Kathmandu Medical College Teaching Hospital, Sinamangal, Kathmandu, Nepal.

** Kanti Children Hospital, Maharajgunj, Kathmandu, Nepal.

Address for correspondence : Dr. Shyam Krishna Maharjan

Kathmandu Medical College Teaching Hospital

Sinamangal, Kathmandu 
occurs as they have increased surface area to body weight ratio, thereby increased evaporation, immature skin losing more water, increased alveolar ventilation increasing water loss via lungs and kidney are less able to concentrate urine. ${ }^{3}$

In order to better define preoperative fasting practices, the house of delegates of American society of Anesthesiologists adopted, in October 1998, practice guidelines for preoperative fasting.

\section{Ingested material}

Minimum fasting period

Clear liquids (water, fruit juices

without pulp, Carbonated beverages,

clear tea, black coffee)

2 hours

Breast milk

4 hours

Infant formula

6 hours

Non human milk

6 hours

Light meal (e.g. toast with clear liquid)

6 hours

These are applied only to healthy patients who are undergoing elective procedure and are not intended to for woman in labor. ${ }^{7}$ The purpose of our randomized study was to compare perioperative outcomes in those with shorter versus longer preoperative fast.

\section{METHODOLOGY}

The ethics committee of the hospital board approved the study protocol and each child's parent gave informed consent. 162 children were under study and divided into two groups. In group one 25 children had glucose water or clear fluid till 2 hours before and 14 children had breast milk at least 4 hours before anaesthesia. The group two 123 children had no solid food from the midnight and no liquid at least 6 hours before the surgery. In these children, the fasting hours was ranging from 6- 16 hours.

Any complications such as regurgitation and vomiting occurring during perioperative period were noted for up to 24 hours by direct observation. The two groups were compared for the complications in different age groups, different fasting hours and type of anaesthesia delivered.

\section{RESULTS}

There were total of 162 children under study. Among them, 34 were infants, 84 were less than 6 years and 44 were more than 6 years of age (Table I). According to fasting hours, 39 were from 2-4 hours group and 123 from traditional group. 74 children got sole intravenous anaesthesia or minor cases, general anaesthesia in 47 children and combination of general or intravenous anaesthesia with regional block in 41 children.

No children had aspiration during perioperative period, which was confirmed by repetative chest auscultation in intraoperative and postoperative period. No children had any sign and symptoms of aspiration during study period. Among the 162 children, 15 had vomiting in post operative ward after 3 hours of transfer from post anaesthesia care unit and all were fully conscious. (Table I)

In infants the incidence of postoperative vomiting was 4 out of 34 (11.76\%); in 1-6 years group, it was 4 out of $84(4.76 \%)$ and in more than 6 years group, it was 7 out of 44 children $(15.9 \%)$. When postoperative vomiting was analyzed in different fasting hours, the incidence was $10.25 \%$ in $2-4$ hours group and $8.94 \%$ in longer fasted group. According to type of anaesthesia delivered, postoperative vomiting was $4.05 \%$ in IVA group; $8.5 \%$ in GA group and $19.5 \%$ in combined group (Table II \& III below).

Postoperative vomiting in group one (shorter fast group) was $10.25 \%$ which was almost same as in group two (longer fast group) i.e. $8.94 \%$. Statistical analysis showed no significant difference $(\mathrm{p}>0.05)$.

Table I : Number of children and complications according to age group 